CLINICAL REPORTS

\title{
A case of insulinoma with peripheral neuropathy
}

\author{
K. S. A. JAYASINGHE \\ M.B.B.S.(Sri Lanka)
}

\author{
A. Nimalasuriya \\ M.D.(Sri Lanka), M.R.C.P. (UK)
}

\author{
K. DHARMADASA \\ M.D.(Sri Lanka), F.R.C.P.(Lond), F.R.C.P.(Edin) \\ Professorial Medical Unit and Department of Medicine, University of Colombo, Kinsey Road, Colombo, Sri \\ Lanka
}

\begin{abstract}
Summary
A 25-year-old woman with chronic neuropsychiatric symptoms, peripheral neuropathy and hypoglycaemia was found to have an islet cell adenoma in the pancreas. Her neuropsychiatric symptoms disappeared following its removal and she partially recovered from her peripheral neuropathy.
\end{abstract}

KEY WORDS: insulinoma, peripheral neuropathy.

\section{Introduction}

Chronic neuropsychiatric symptoms attributed to hypoglycaemia are a well known presentation of insulinoma. Peripheral neuropathy has been rarely reported in such patients. The first report of a case suggestive of peripheral neuropathy with hypoglycaemia and an insulinoma at laparatomy was described by Silfverskiold (1946) and subsequently 21 cases have been reported in the literature (Danta, 1969). A case of amyotrophy associated with an insulinoma has been described by Williams (1955).

We report a case of an insulinoma presenting with a chronic neuropsychiatric syndrome, associated with sensorimotor peripheral neuropathy which recovered partially following surgical removal of the insulinoma.

\section{Case report}

A 25-year-old stenography student was admitted to the neurosurgical unit of the General Hospital in December 1980 with a history of 3 episodes of altered consiousness, dysarthria and difficulty in walking of about one year duration. She was subsequently referred to our unit for investigation and exclusion of an insulinoma.

The first attack was observed in December 1979. She had 2 further attacks in March and April 1980.
Each attack came after the patient missed a meal and was characterized by gradual onset of disorientation and confusion. These attacks were aborted by having a glass of sweetened milk or food. She had no sweating or seizures and was amnesic of the episodes.

From April 1980 she had drowsiness and confusion on waking which would improve with her morning cup of sweetened milk. She also noticed a progressive weakness and numbness of her extremities from April which progressively worsened. She had difficulty in sitting up from the squatting position and had to give up typing due to the weakness. From May 1980 she became dysarthric. There was no family history of endocrine disease or history of exposure to weed killers, lead or alcohol.

On examination, she was emaciated and rational, but she became confused on overnight fasting. In addition to the dysarthria, she had mild wasting of the muscles of both her arms and forearms with gross wasting of small muscles of the hands. She also had wasting of her quadriceps. No fasciculations were observed. There was distal weakness of her upper limbs and distal and proximal weakness of her lower limbs. She had glove and stocking type of sensory loss in the hands and feet. Vibration sense was absent at the ankles. The deep tendon jerks were normal except for absent ankle jerks.

Random blood glucose levels were $1.66 \mathrm{mmol} /$ litre, $1.39 \mathrm{mmol} /$ litre, with a blood glucose of 0.72 $\mathrm{mmol} /$ litre after overnight fasting.

Routine haematological and biochemical investigations including the erythrocyte sedimentation rate were normal. No LE cells were detected. Urinary porphobilinogen and porphyrins were not detected. Electroencephalogram revealed irregular and slow waves, without significant asymmetry, compatible with metabolic changes affecting the brain. Coeliac axis arteriogram did not reveal a vascular lesion in 
the pancreas. Electromyographic studies showed a reduction in the recruitment pattern with high proportion of highly polyphasic units of low-normal voltage. Motor conduction velocities were grossly delayed, (Wrist-lst dorsal interossei 20 millisecs. Elbow-1st dorsal interossei 32 millisecs.) indicating evidence of a very severe peripheral neuropathy.

Operation revealed a well encapsulated lobulated tumour $6 \mathrm{~cm} \times 5 \mathrm{~cm}$ in size at the junction of the body and tail of the pancreas. No other masses or liver nodules or ascites was seen. The histology was reported as compatible with a functioning islet cell adenoma, showing a predominantly trabecular and ribbon-like pattern. It was well demarcated from the surrounding tissue by a fibrous capsule. She made an uneventful postoperative recovery and her neuropsychiatric manifestations and sensory neuropathy disappeared. The fasting blood glucose level was found to be $3.9 \mathrm{mmol} /$ litre on discharge 2 months after surgery. Review 6 months after surgery revealed that the weakness and dysarthria had improved and she had resumed work as a typist.

\section{Discussion}

Insulinoma is a functioning endocrine tumour arising from beta cells of the pancreas. The neuroglycopenia of an insulinoma gives symptoms chiefly neuropsychiatric in nature such as drowsiness, syncope, loss of emotional control, irrational behaviour, vertigo, coma and convulsions.

Pathologically, there is selective nerve cell damage of the cortex, sometimes focal, but more usually laminar with an emphasis on the third and fifth layers. Diffuse demyelination and fibrillary gliosis with normal myelin staining have also been described. The hippocampi are also invariably involved, but the subcortical structures, cerebellum and brainstem are mildly involved. The spinal cord and peripheral nerves are spared in most cases, but in those with involvement, the pathology in the peripheral nerves has yet to be definitely identified (Blackwood and Corsellis, 1976). Lambert, Mulder and Bastron (1960) have suggested that it is due to peripheral neuropathy, while Tom and Richardson (1951) described a case where the arterior horn cells of the spinal cord were damaged. Bradley (1974) has described degeneration of the cell body of the axom within the spinal cord and dorsal root.

Although the pathogenesis of the neuropsychiatrfe manifestations are known, the pathogenesis of the peripheral nerve manifestations is unknown. Energy metabolism in peripheral nerves is both quantita: tively and qualitatively different from that in the brain and the peripheral nerves are less dependent o glucose (Abood, 1960). On the basis of this, it is to be expected that, with damage to the peripheral nerves due to hypoglycaemia, the affliction of the centrat nervous system should be more pronounced. Of the 24 cases with peripheral neuropathy reviewed bab Danta (1969), signs indicative of sustained damage if the brain were present in only 7 patients, which is ng in keeping with the above view. The possibility of insulin or other substances released by the tumoux causing the peripheral nerve damage exists. The are no studies relating the insulin concentrations $t \theta$ the manifestation of peripheral neuropathy. More studies are needed to determine the aetiology of the peripheral neuropathy associated with insulinoma..

\section{Acknowledgments}

We wish to thank Dr L. Gunasekera F.R.C.S. neuro-surga General Hospital, Colombo and Dr S. J. Stephen M.S. F.ק承 thoracic surgeon, General Hospital, Colombo.

\section{References}

ABOoD, L.G. (1960) Handbook of Physiology: I p. 1815. American Physiology Society, Washington.

BlaCKWOOD, M.P. \& CORSEllis, J.A.N. (1976) Greenfield's Neure pathology 3rd edn., p. 74. Edward Arnold Ltd., London.

BRADLEY, W.G. (1974) Disorders of peripheral nerves p. 173 Blackwell Scientific Publications, Oxford and London.

DANTA, G. (1969) Hypoglycaemic peripheral neuropathy. Archive of Neurology, 21, 121.

LAMBERT, E.H., MUlder, D.W. \& BASTRON, J.A. (1960) Regener民 tion of peripheral nerves with hyperinsulin neuropathy. Neur $\log y, 10,851$.

SILFVERSKIOLD, B.P. (1946) Polyneuritis hypoglycaemia, late per. pheral paresis after hypoglycaemic attacks in two insulinomga patients Acta medica Scandinavica, 125, 502.

TOM, M.I. \& RICHARDSON, J.C. (1951) Hypoglycaemia from isletcell tumour of pancreas with amyotrophy and cerebrospinal nerf cell changes; a case report. Journal of Neuropathology, 10, 57. I

WILLIAMS, C.J. (1955) Amyotrophy due to hypoglycaemia. Briti Medical Journal, 1, 707.

(Accepted 17th June 1982) 